Cite this: J. Mater. Chem. A, 2014, 2, 7723

Received 21st February 2014 Accepted 11th April 2014

DOI: $10.1039 / c 4 t a 00907 j$

www.rsc.org/MaterialsA

\section{Rapid, microwave-assisted thermal polymerization of poly(ethylene glycol) diacrylate-supported ionogels $\uparrow$}

\author{
Adam F. Visentin, Tingyi Dong, Jonathan Poli and Matthew J. Panzer*
}

\begin{abstract}
Current options for forming ionic liquid-based solid electrolytes (ionogels) often involve slow processes; however, by leveraging the inherent ability of the ionic liquid to harness the energy of microwave irradiation, a gel-forming, thermal polymerization can be achieved in a matter of seconds. The resulting ionogel electrolyte exhibits comparable electrical and mechanical performance to gels produced via conventional fabrication techniques.
\end{abstract}

Solid electrolytes comprised mainly of nonvolatile, room temperature ionic liquids with a small amount of a solid supporting scaffold material, termed ionogels, are currently being investigated for a variety of electronic device applications, including: transistors, ${ }^{\mathbf{1 , 2}}$ electrochemical capacitors, ${ }^{3-6}$ batteries,,$^{7,8}$ and solar cells. ${ }^{8}$ Ionogels offer the many benefits of ionic liquid electrolytes (nonvolatility, ionic conductivity, wide electrochemical window) in a leak-proof, solid form. ${ }^{\mathbf{9 , 1 0}}$ In this report, a simple microwave heating method that can be used to realize the rapid formation of an ionogel (less than $30 \mathrm{~s}$ ) is presented. The great utility of microwave technology has already been demonstrated in the use of ionic liquids as solvents for polymer synthesis. ${ }^{11,12,13}$ The ability of a solvent to absorb energy via microwave irradiation allows for a swift temperature rise in the liquid; this effect can be enhanced for solvents that exhibit ionic conductivity. ${ }^{14,15}$ Among the many advantages of microwave heating are decreased reaction times and an even heat distribution in the reaction medium, i.e. no wall effects. ${ }^{16}$ Microwave heating of several common ionic liquids has been well-studied; ${ }^{\mathbf{1 4 , 1 5 , 1 7 - 1 9}}$ however, to date, there have been no known reports of the use of microwave irradiation to create a free-standing, solid ionogel electrolyte.

Although several ionogel fabrication strategies have been reported in recent years, most of these require a processing time measured in several minutes to hours to achieve a gel (Fig. 1). In

Department of Chemical \& Biological Engineering, Tufts University, 4 Colby St., Medford, Massachusetts 02155, USA. E-mail: matthew.panzer@tufts.edu

$\uparrow$ Electronic supplementary information (ESI) available: Experimental procedure and characterization details. See DOI: 10.1039/c4ta00907j cases where drying or vacuum treatment steps are employed to remove volatile co-solvents or other gelation reaction byproducts, total gel formation times can be several days. Common ionogel fabrication methods include: use of a co-solvent to blend in a polymer support, ${ }^{3,5,20-22}$ initiation of a spontaneous sol-gel reaction to produce an inorganic support, ${ }^{23-26}$ stirring in an assembly of fumed silica particles, ${ }^{27-29}$ and either UV-initiated $^{30-33}$ or thermally-initiated ${ }^{34-40}$ polymerization/crosslinking of a reactive monomer inside the ionic liquid. Additionally, chemical vapor deposition and alternative polymerization strategies that require on the order of minutes have been reported. ${ }^{\mathbf{4 1 4 2}}$ UV-initiated polymerizations can also be employed to create ionogels in several minutes according to some reports $;^{30-32}$ however, this is still not as rapid as is demonstrated here for microwave processing (Fig. 1), which can be as fast as 10-25 s.

The microwave-enabled ionogel fabrication approach described here enables one to dramatically reduce the time

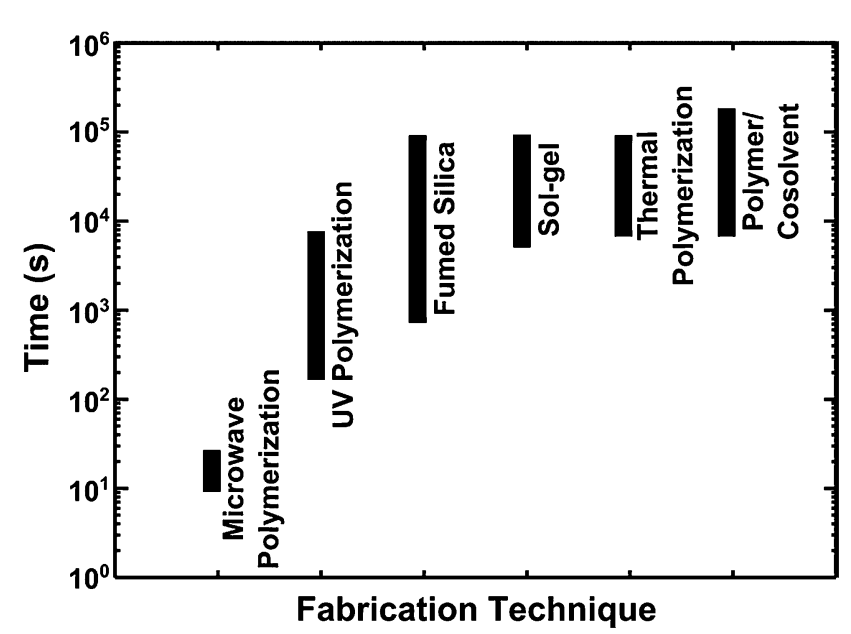

Fig. 1 A comparison of the ranges of typical ionogel formation times (in seconds) reported for various fabrication techniques: microwaveassisted polymerization (this work), UV-initiated polymerization, ${ }^{30-33}$ fumed silica addition, ${ }^{27-29}$ sol-gel scaffold, ${ }^{23-26}$ thermally-initiated polymerization, ${ }^{34-40}$ and polymer/cosolvent blends.,5,20,21 
typically required for a thermally-initiated free radical polymerization/crosslinking reaction inside the ionic liquid due to extremely rapid heating. The ability to rapidly heat a liquid via microwave irradiation is driven by interactions between the wave and a molecular dipole. When a molecule absorbs microwave energy, its dipole shifts to align with the electric field; this shift creates friction between adjacent molecules, resulting in the dissipation of some energy as heat. The predicted rate of temperature rise can be expressed as: ${ }^{16}$

$$
\frac{\Delta T}{t}=\frac{\omega \varepsilon_{0} \varepsilon^{\prime \prime}|E|^{2}}{\rho C_{\mathrm{P}}}
$$

where $\Delta T / t$ is the time rate of temperature change, $\omega$ is the angular frequency of the microwave radiation, $\varepsilon_{0}$ is the vacuum permittivity, $\varepsilon^{\prime \prime}$ the imaginary part of the liquid complex relative permittivity (evaluated at frequency $\omega$ ), $|E|$ is the electromagnetic field strength, $\rho$ is the liquid density, and $C_{\mathrm{P}}$ is the liquid specific heat capacity. As the dielectric loss tangent is directly proportional to $\varepsilon^{\prime \prime}$, its magnitude reflects the relative efficiency by which electromagnetic energy can be converted into heat. It should be noted that commercial domestic microwave ovens operate at a frequency of $2.45 \mathrm{GHz}$. Table 1 summarizes three important parameters that determine the expected temperature rise for one of the most commonly studied ionic liquids, 1-ethyl3-methylimidazolium-bis(trifluoromethanesulfonyl)imide (EMI TFSI), as well as for water.

Using eqn (1), it follows that the predicted microwave heating rate of EMI TFSI is approximately $40 \%$ higher than that of water for a given electric field strength. Although its $\varepsilon^{\prime \prime}$ value at $2.45 \mathrm{GHz}$ is slightly lower than that of water, the smaller densityspecific heat capacity product of EMI TFSI facilitates a greater temperature rise per amount of microwave energy absorbed. For ionic liquids, one major advantage of using microwave heating is that their ionic conductivity facilitates strong electric dipole perturbations by the applied field. Thus, ionic liquids respond strongly to low frequency microwaves, such as those used in domestic microwaves. The result is a dramatic rate of ionic liquid temperature rise, which has been reported to exceed $10^{\circ} \mathrm{C} \mathrm{s}^{-1} .{ }^{17}$ Combined with these extremely rapid heating rates, the nonvolatile nature of ionic liquids allows for thermally-driven reactions to occur swiftly at high temperatures. A general hallmark of microwave heating as a technique is the lack of physical contact required between the heat source and the liquid; bypassing this conventional thermal conduction step can eliminate the time required for heat transfer within the reaction volume. The combination of all these factors highlights the outstanding ability to perform a rapid thermal polymerization of a reactive monomer within an ionic liquid to form an ionogel.

Table 1 Parameters relevant to predicted microwave heating rates

\begin{tabular}{lcll}
\hline Liquid & $\varepsilon^{\prime \prime}$ at $2.45 \mathrm{GHz}$ & $\rho\left(\mathrm{g} \mathrm{cm}^{-3}\right)$ & $C_{\mathrm{P}}\left(\mathrm{J} \mathrm{g}^{-1}{ }^{\circ} \mathrm{C}^{-1}\right)$ \\
\hline EMI TFSI & $8^{a}$ & $1.52^{c}$ & $1.30^{c}$ \\
Water & $12^{b}$ & 1.00 & 4.19
\end{tabular}

${ }^{a}$ Ref. $18 .{ }^{b}$ Ref. $16 .{ }^{c}$ Ref. 43.
In order to demonstrate the feasibility of microwave-assisted ionogel formation, EMI TFSI was selected as the ionic liquid solvent. While a detailed procedure can be found in the ESI, $\dagger$ a brief description follows here. A solution containing requisite amounts of the ionic liquid, AIBN (thermal initiator) and poly(ethylene glycol) diacrylate (PEGDA) monomer (575 g $\mathrm{mol}^{-1}$ ) were prepared. The solution contained approximately $25 \mathrm{wt} \%$ PEGDA, as lower scaffold amounts did not reliably produce free-standing gels via the microwave-assisted approach. Solutions were sonicated to aid in mixing and to degas. Liquid precursor solution was heated inside a commercial domestic microwave oven using "on" pulses of $5 \mathrm{~s}$ duration and a total heating time of $10 \mathrm{~s}$ to $25 \mathrm{~s}$. A pulsing strategy was employed in order to prevent overheating and subsequent thermal decomposition of the solution. All of the preceding steps were conducted under ambient laboratory conditions. The resulting ionogel material is an optically clear, freestanding solid.

The ionogel was found to contain a highly polymerized scaffold, as determined by FTIR spectroscopy (Fig. 2). The acrylate double bond $\left(\mathrm{CH}_{2}=\mathrm{CH}\right)$ of the PEGDA monomer exhibits two distinct peaks in the FTIR spectra, highlighted by the vertical dotted lines in Fig. 2 at $810 \mathrm{~cm}^{-1}$ and $1410 \mathrm{~cm}^{-1}$. Comparison of the three spectra shown in Fig. 2 reveals the absence of these peaks for the ionogel, as well as for neat EMI TFSI.

The mechanical and electrical responses of PEGDA-supported EMI TFSI ionogels fabricated via microwave-assisted thermal polymerization were characterized in order to validate this method as a means of producing ionogels with properties similar to those produced by a previously-reported technique (UV-initiated polymerization/crosslinking). ${ }^{27}$ The ionic conductivity of the ionogel was measured to be $3.3 \mathrm{mS} \mathrm{cm}^{-1}$ by AC impedance spectroscopy (Fig. S1 $\dagger$ ); the corresponding value for EMI TFSI was $9.7 \mathrm{mS} \mathrm{cm}^{-1}$. A reduction in ionic conductivity of the ionogel compared to the neat ionic liquid is typically observed upon the addition of the scaffold material..$^{37,39,40}$

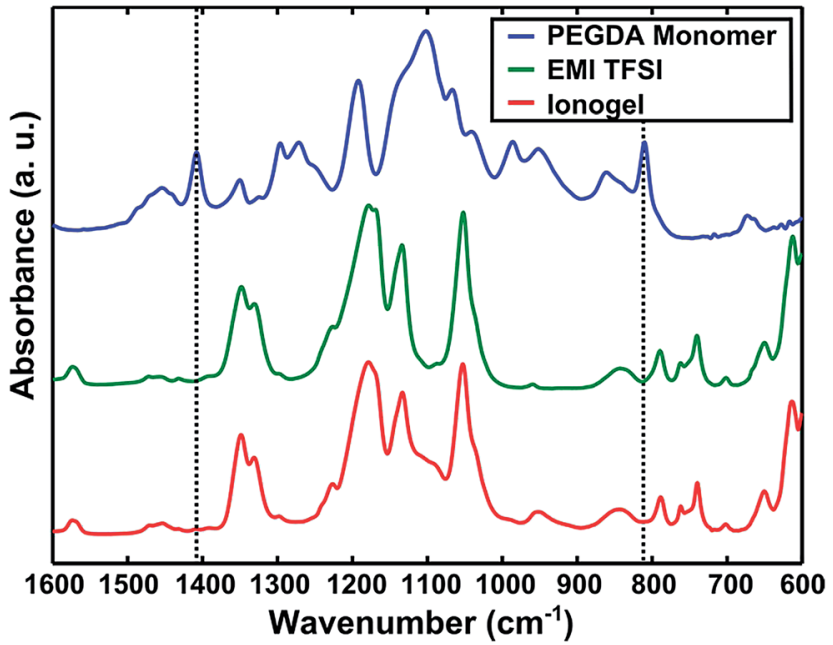

Fig. 2 FTIR spectra of the PEGDA monomer (blue), neat EMI TFSI (green), and a microwave-synthesized ionogel (red). 
Compared to the ionic conductivity of an ionogel with a UVpolymerized scaffold of the same PEGDA mass fraction $(\sim 2 \mathrm{mS}$ $\mathrm{cm}^{-1}$ ), ${ }^{30}$ the conductivity was $\sim 65 \%$ greater for the microwavesynthesized ionogel.

Mechanical characterization of the ionogel was performed via compression testing. Compression was chosen over tensile testing because it is believed to be more representative of the type of deformation an ionogel would experience in many device applications (e.g. normal force applied to a thin film battery). Compressive loading and unloading data for a microwave-synthesized ionogel are shown in Fig. 3.

The stress-strain data indicate highly elastic deformation up to at least $15 \%$ strain, as confirmed by minimal hysteresis between the loading and unloading curves. These microwavesynthesized PEGDA ionogels (25 wt\% scaffold) exhibit Young's moduli of approximately $200 \mathrm{kPa}$ to $500 \mathrm{kPa}(5-10 \%$ strain regime), based on measurements made using several samples fabricated under identical conditions. It should be noted that the modulus value of a UV-cured ionogel containing $25 \mathrm{wt} \%$ PEGDA is approximately $6 \mathrm{MPa},{ }^{30}$ which is at least one order of magnitude larger by comparison. This difference may be explained by an enhanced degree of interchain crosslinking for the UV-cured PEGDA scaffold in EMI TFSI compared to the scaffold formed under extremely rapid heating via the microwave-assisted thermal polymerization conditions employed here. This conclusion is also supported by the observation that PEGDA contents lower than $25 \mathrm{wt} \%$ did not result in reliable microwave-synthesized ionogels using the procedure outlined here, while the minimum amount of PEGDA needed to form a free-standing ionogel by the UV-curing approach is only $\sim 5$ wt $\%{ }^{30,44}$ While beyond the scope of this present study, the exact temperature versus time heating profile is also believed to play an important role in the scaffold polymerization. In addition to any changes in the initiation/propagation/termination rate constants with temperature, it has been previously found that the ionic liquid viscosity (which also varies strongly with

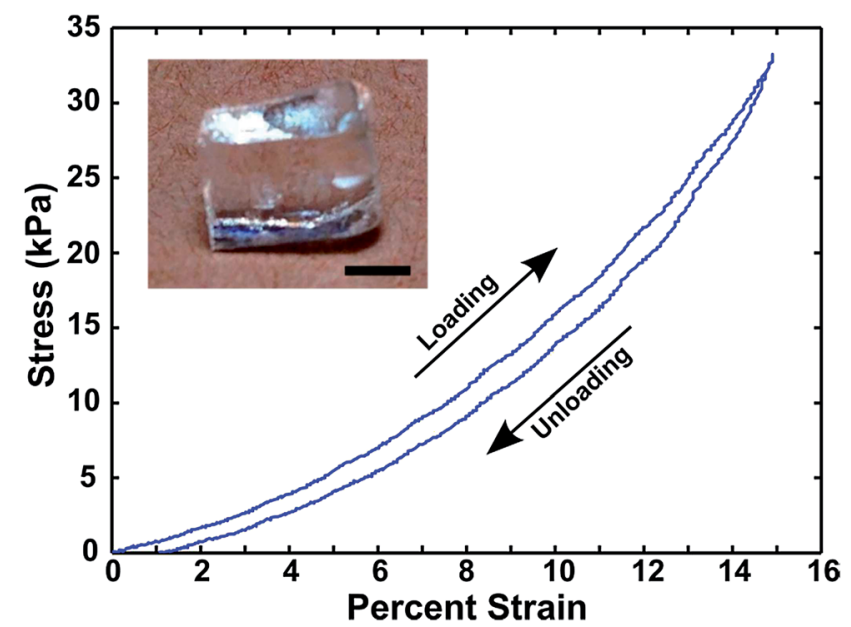

Fig. 3 Compressive stress-strain characteristics of a microwavesynthesized ionogel, which exhibits elastic behavior up to $15 \%$ strain. Inset: photograph of a representative microwave-synthesized ionogel (scale bar $=2 \mathrm{~mm}$ ).

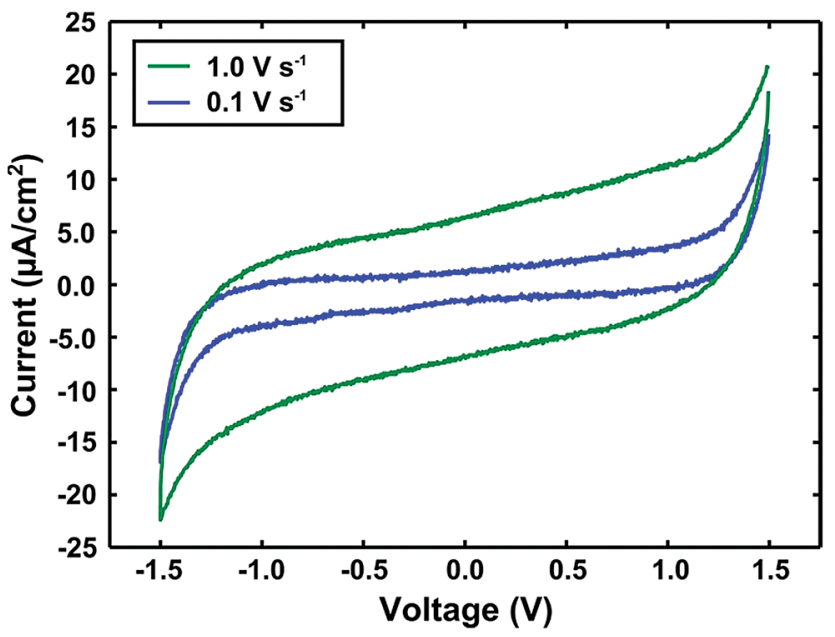

Fig. 4 Cyclic voltammograms of a microwave-synthesized ionogel between glassy carbon electrodes at two different voltage sweep rates.

temperature) can be a determining factor in the scaffold formation of UV-polymerized PEGDA ionogels, where the moduli are reflective of the effective crosslink densities. ${ }^{\mathbf{3 0 4 4}}$

The capacitive nature of the microwave-synthesized ionogel was verified by two-electrode cyclic voltammetry (CV). Fig. 4 displays CV spectra recorded at two different voltage sweep rates for an ionogel sandwiched between glassy carbon electrodes. An approximate specific device capacitance of $6 \mu \mathrm{F} \mathrm{cm} \mathrm{cm}^{-2}$ can be extracted from the $\mathrm{CV}$ data (by dividing the average $0 \mathrm{~V}$ bias current magnitude by the sweep rate), which is consistent with such devices. ${ }^{\mathbf{3 0 , 4 4}}$ The slightly sloped shape of the faster scan rate spectrum can be attributed to resistance in both the ionogel material and the testing setup. Importantly, the lack of redox current peaks near $\pm 1 \mathrm{~V}$ indicates that there is no significant amount of water present, despite fabrication and testing under ambient conditions. Taken collectively, these observations confirm that the microwave-synthesized ionogel performs in a manner that is largely consistent with similar gels produced using one of the standard ionogel fabrication methods (UVcuring).

\section{Conclusions}

Microwave heating has been successfully used to fabricate an ionogel for the first time. Extremely rapid heating due to the ability of the ionic liquid to absorb microwave energy has enabled gelation in as little as $10 \mathrm{~s}$. This timescale is significantly swifter than any other known method for producing ionogels. EMI TFSI has been employed as a model ionic liquid to demonstrate the feasibility of rapid thermal polymerization of PEGDA within the liquid volume, forming a solid, supporting scaffold. Microwave-synthesized ionogels exhibit electrical and mechanical properties that are comparable to those of ionogels fabricated by other methods. Further control of the microwaveassisted heating profile may lead to even faster fabrication times for high performance, solid ionogel electrolytes. 


\section{Acknowledgements}

This work is financially supported by the National Science Foundation (award no. ECCS-1201935) and Tufts University.

\section{Notes and references}

1 J. Lee, M. J. Panzer, Y. He, T. P. Lodge and C. D. Frisbie, J. Am. Chem. Soc., 2007, 129, 4532.

2 J. Lee, L. G. Kaake, J. H. Cho, X. Zhu, T. P. Lodge and C. D. Frisbie, J. Phys. Chem. C, 2009, 113, 8972.

3 C. Liew, S. Ramesh and A. K. Arof, Int. J. Hydrogen Energy, 2014, 39, 2917.

4 G. P. Pandey and S. A. Hashmi, J. Mater. Chem. A, 2013, 1, 3372.

5 G. P. Pandey, S. A. Hashmi and Y. Kumar, Energy Fuels, 2010, 24, 6644 .

6 C. Yang, J. B. Ju, J. K. Lee, W. I. Cho and B. W. Cho, Electrochim. Acta, 2005, 50, 1813.

7 J. L. Bideau, J. Ducros, P. Soudan and D. Guyomard, Adv. Funct. Mater., 2011, 21, 4073.

8 J. L. Bideau, L. Viau and A. Vioux, Chem. Soc. Rev., 2011, 40, 907.

9 T. Ueki and M. Watanabe, Macromolecules, 2008, 41, 3740. 10 J. Lu, F. Yan and J. Texter, Prog. Polym. Sci., 2009, 34, 431. 11 S. Mallakpour and Z. Rafiee, Prog. Polym. Sci., 2011, 36, 1754. 12 K. Kempe, C. R. Becer and U. S. Schubert, Macromolecules, 2011, 44, 5825.

13 J. Lu, F. Yan and J. Texter, Prog. Polym. Sci., 2009, 34, 431.

14 E. I. Izgorodina, M. Forsyth and D. R. MacFarlane, Phys. Chem. Chem. Phys., 2009, 11, 2452.

$15 \mathrm{~J}$. Hoffmann, M. Nuchter, B. Ondruschka and P. Wassercheid, Green Chem., 2003, 5, 296.

16 C. Gabriel, S. Gabriel, E. H. Grant, B. S. J. Halstead and D. M. Mingos, Chem. Soc. Rev., 1998, 27, 213.

17 D. Obermayer and C. O. Kappe, Org. Biomol. Chem., 2010, 8, 114.

18 G. Dimitrakis, I. J. Villar-Garcia, E. Lester, P. Licence and S. Kingman, Phys. Chem. Chem. Phys., 2008, 10, 2947.

19 S. Horikoshi, T. Hamamura, M. Kajitani, M. YoshizawaFujita and N. Serpone, Org. Process Res. Dev., 2008, 12, 1089.

20 S. Zhang, K. H. Lee, C. D. Frisbie and T. P. Lodge, Macromolecules, 2011, 44, 940.

21 K. H. Lee, S. Zhang, T. P. Lodge and C. D. Frisbie, J. Phys. Chem. B, 2011, 115, 3315.

22 Y. Kitazawa, T. Ueki, K. Niitsuma, S. Imaizumi, T. P. Lodge and M. Watanabe, Soft Matter, 2012, 8, 8067.
23 A. I. Horowitz and M. J. Panzer, J. Mater. Chem., 2012, 22, 16534.

24 T. Mizumo, T. Watanabe and H. Ohno, Polym. J., 2008, 40, 1099.

25 M. Neouze, J. L. Bideau, F. Leroux and A. Vioux, Chem. Commun., 2005, 1082.

26 Z. Li, H. Liu, Y. Liu, P. He and J. Li, J. Phys. Chem. B, 2004, 108, 17512.

27 K. Ueno, K. Hata, T. Katakabe, M. Kondoh and M. Watanabe, J. Phys. Chem. B, 2008, 112, 9013.

28 J. Nordstrom, L. Aguilera and A. Matic, Langmuir, 2012, 28, 4080.

29 K. Ueno, S. Imaizumi, K. Hata and M. Watanabe, Langmuir, 2009, 25, 825.

30 A. F. Visentin and M. J. Panzer, ACS Appl. Mater. Interfaces, 2012, 4, 2836.

31 I. Stepniak and E. Andrzejewska, Electrochim. Acta, 2009, 54, 5660.

32 J. T. Delaney Jr, A. R. Liberski, J. Perelaer and U. Schubert, Macromol. Rapid Commun., 2010, 31, 1970.

33 C. Yang, W. I. Cho, J. K. Lee, H. Rhee and B. W. Cho, Electrochem. Solid-State Lett., 2005, 8, A91.

34 J. F. Stanzione, R. E. Jensen, P. J. Costanzo and G. R. Palmese, ACS Appl. Mater. Interfaces, 2012, 4, 6142.

35 Y. S. Vygodskii, D. A. Sapozhinkov, A. S. Shaplov, E. I. Lozinskaya, N. V. Ignat'ev, M. Schulte, P. S. Vlasov and I. A. Malyshkina, Polym. J., 2011, 43, 126.

36 Y. Gu, S. Zhang, L. Martinetti, K. H. Lee, L. D. McIntosh, C. D. Frisbie and T. P. Lodge, J. Am. Chem. Soc., 2013, 135, 9652.

37 M. A. B. H. Susan, T. Kaneko, A. Noda and M. Watanabe, J. Am. Chem. Soc., 2005, 127, 4976.

38 Y. Isshiki, M. Nakamura, S. Tabata, K. Dokko and M. Watanabe, Polym. Adv. Technol., 2011, 22, 1254.

39 K. Matsumoto, S. Sogabe and T. Endo, J. Polym. Sci., Part A: Polym. Chem., 2010, 48, 4515.

40 J. Jiang, D. Gao, Z. Li and G. Su, React. Funct. Polym., 2006, 66, 1141.

41 K. Fujii, H. Asai, T. Ueki, T. Sakai, S. Imaizumi, U. Chung, M. Watanabe and M. Shibayama, Soft Matter, 2012, 8, 1756.

42 R. J. Frank-Finney, L. C. Bradley and M. Gupta, Macromolecules, 2013, 46, 6852.

43 Y. U. Paulechka, A. G. Kabo, A. V. Blokhin, G. J. Kabo and M. P. Shevelyova, J. Chem. Eng. Data, 2010, 55, 2719.

44 A. F. Visentin, S. Alimena and M. J. Panzer, ChemElectroChem, 2014, 1, 718. 